\title{
MIOPATIA OCULAR DESCENDENTE
}

\author{
Marcos R. G. de Freitas * \\ Osvaldo J. M. Nascimento **
}

O estudo das afecções dos músculos elevadores das pálpebras e da motilidade extrinseca dos olhos tem sido feito desde longa data. Inicialmente pensavam os autores que isso fosse devido a degeneração dos núcleos óculo-motores. Fuchs, em 1890, colocou em dúvida tal afirmativa, já suspeitando de um processo miopático. Taylor ${ }^{19}$, em 1915, estudou uma família na qual havia ptose palpebral e paralisia vagoglossofaringea, 'que também atribuiu à degeneração nuclear no tronco cerebral. Em 1946, Sandifer ${ }^{17}$ e, em 1951, Kiloh e Nevin ${ }^{10}$ atribuíram à degeneração primária das fibras musculares a origem de tais of talmoplegias. Schwarz e Liu ${ }^{18}$, em 1954, fizeram apurado estudo anatomopatológico de paciente com ptose palpebral bilateral e oftalmoplegia extrínseca completa, confirmando o caráter miogênico.

A afecção estudada por Kiloh e Nevin ${ }^{10}$, chamada miopatia ocular, teria seu início no adulto jovem, de evolver lento e progressivo, levando à ptose palpebral bilateral e à oftalmoplegia extrínseca completa. Já a entidade primeiramente descrita por Taylor ${ }^{19}$ ocorreria após a quinta década da vida, quase sempre hereditária do tipo autossômico dominante, caracterizada por ptose palpebral bilateral, eventualmente paralisia dos músculos extrinsecos do olho e também comprometimento da musculatura faríngea. Foi denominada miopatia ocular descendente (Lees e Liversedge ${ }^{11}$ ) ou miopatia óculo-faríngea (Victor, Hayes e Adams ${ }^{21}$ ). Assim, a miopatia ocular descendente é tida por alguns autores (Bray e col. ${ }^{4}$, Walton ${ }^{23,}{ }^{24}$ ) como entidade autônoma, pelo inicio tardio e pelo caráter familial.

O nosso propósito é relatar caso de paciente jovem do sexo feminino, apresentando ptose palpebral bilateral, paralisia dos músculos extrínsecos dos olhos e da musculatura vago-glossofaríngea, de evolver progressivo, tendo inicio aos 18 anos de idade. Esperamos assim poder contribuir para o estudo desta rara afecção, tentando melhor situá-la entre as miopatias primárias.

\footnotetext{
* Responsável pela Disciplina de Neurologia junto à Chefia do Departamento de Medicina Clínica da Universidade Federal Fluminense, Auxiliar de Ensino de Neurologia da UFRJ (Instituto de Neurologia Deolindo Couto), Serviço do Prof. Bernardo Couto; * Auxiliar de Ensino da Disciplina de Neurologia da Universidade Federal Fluminense, Bolsista do Conselho de Pesquisas da UFRJ no Instituto de Neurologia Deolindo Couto (Serviço de Neuropatologia do Prof. Alexandre Alencar).
} 


\section{OBSERVAÇÃO}

V. V. C. (Reg. 85649, Hospital Universitário Antonio Pedro), 23 anos, sexo feminino, branca, professora, internada em 09-07-1973. Início da afecção há 5 anos, quando passou a observar queda de pálpebras bilateralmente, de início lento e progressivo, sendo que, em dois anos, havia ptose palpebral. Niega diplopia durante a evolução do quadro. Há dois anos, verificou dificuldade para deglutir e para falar que vem-se agravando lentamente. Fez tratamento com mestinon e corticosteróides, sem resultado. Antecedentes pessoais — Viroses comuns na infância e amigdalectomia aos 9 anos. Antecedentes familiares - Nega casos semelhantes na familia. Exame neurológico - Diminuição da força de abdução dos membros superiores. Hipotonia generalizada. Coordenação normal. Reflexos cutâneo-abdominais presentes. Reflexos cutâneo-plantares em flexão. Reflexos bicipitais abolidos; demais reflexos presentes e simétricos. Sensibilidade normal. Eusência de fenômeno miotônico. Nervos cranianos - Ptose palpebral bilateral (Fig. 1). Abolição dos movimentos do globo ocular na horizontalidade e na verticalidade. Reflexos pupilares conservados. Diminuição da força de contração dos orbiculares das pálpebras. Paresia bilateral do vêu do paladar com disfonia e disfagia. Demais nervos cranianos normais. A prova da prostigmina não alterou o quadro. Exames complementares - Hemograma normal. Glicose $94 \mathrm{mg} \%$; uréia $33 \mathrm{mg} \%$; creatinima $0,9 \mathrm{mg} \%$; colesterol $199 \mathrm{mg} \%$; sódio $142 \mathrm{mEq} / 1$; potássio $4,2 \mathrm{mEq} / 1$; cloretos $104 \mathrm{mEq} / 1$; bicarbonato $25 \mathrm{mEq} / 1$. Reação do VDRL negativa. Eletroforese de proteinas séricas: proteinas totais $7 \mathrm{~g} \%$; albumina $44 \%$; alfa - globulina $6,5 \%$, alfa - globulina $8,3 \%$; beta globulina $17,4 \%$, gama globulina 23,2\%. TGO $10 \mathrm{U}$. Dehidrogenase lática 84 UI; creatino-fosfoquinase $0,5 \mathrm{U}$ sigma. Urina sem alterações. Eletrocardiograma: arritmia sinusal, 70/min., dentro dos limites normais. Radiografias do cranio normais; radiografia de tórax normal. Trânsito esofagiano: fenômeno de refluxo faringo-nasal com repleção do epifaringe e fossas nasais. Retardo do esvaziamento do hipofaringe (Fig. 2) com estase do contraste nas valéculas (Dr. Oswaldo Fernandes). Eletromiograma dos orbiculares das pálpebras (Fig. 3) - Potenciais de inserção normais; em repouco silêncio elétrico; durante a contração muscular: U. M. de baixa amplitude e duraçāo inferior a $10 \mathrm{~m} / \mathrm{seg}$; discrepância entre a intensidade de contracão muscular (contração fraca) e o número. excessivo de potenciais unitários; exagero de somação espacial. Conclusão: mui sugestivo de processo miogênico (Dr. Fernando Sepúlveda). Biópsia muscular do elevador da pálpebra direita e reto externo direito (Fig. 4) - Músculo apresentando fragmentação das estriaçōes transversais, fibras de diâmetros diferentes, em vários graus de atrofia, mostrando degeneração do sarcoplasma; tecido fibro-adiposo bem mais rico que o habitual, mostrando certa correspondência em intensidade com as áreas de maior atrofia (substituição fibro-adiposa); núcleos picnóticos são observados. Conclui-se, portanto, por um quadro de distrofia muscular.

\section{COMENTARIOS}

Trata-se, portanto, de paciente com 23 anos de idade e do sexo feminino, com ptose palpebral, oftalmoplegia extrinseca completa bilateral e paresia da musculatura vago-glossofaringea, de evolver progressivo em 5 anos. O eletromiograma mostrou padrão miogênico e a biópsia muscular foi compatível com miopatia primária. Não há casos semelhantes na família e a prova da prostigmina foi negativa. Com estes elementos, chegamos à conclusão de se tratar de afecção muscular primária envolvendo os músculos elevadores das pálpebras, a musculatura extrínseca dos olhos e os músculos faríngeos.

As afecções primárias que comprometem a musculatura ocular são hoje divididas por alguns autores em duas entidades distintas: a miopatia ocular e a miopatia ocular descendente (miopatia óculo-faríngea). 


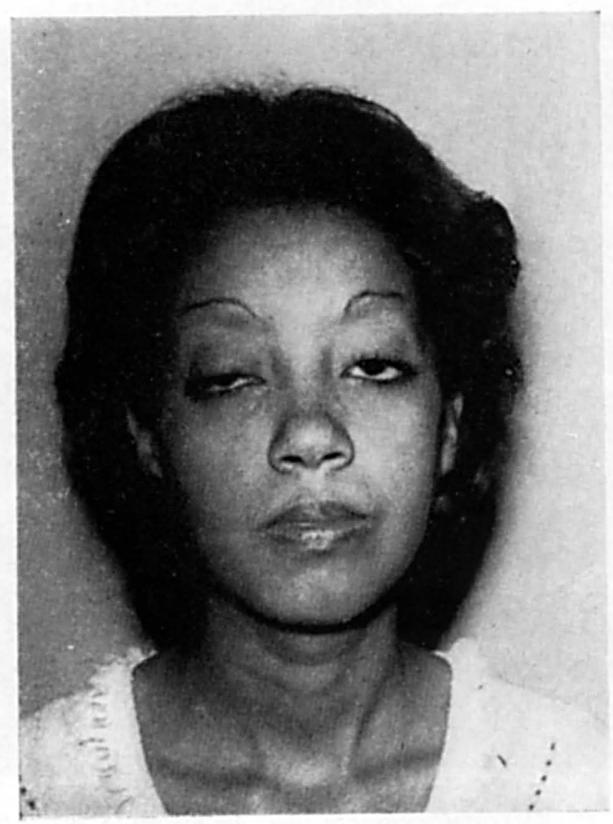

Fig. 1 - Caso V. V. C. Ptose palpebral bilateral.
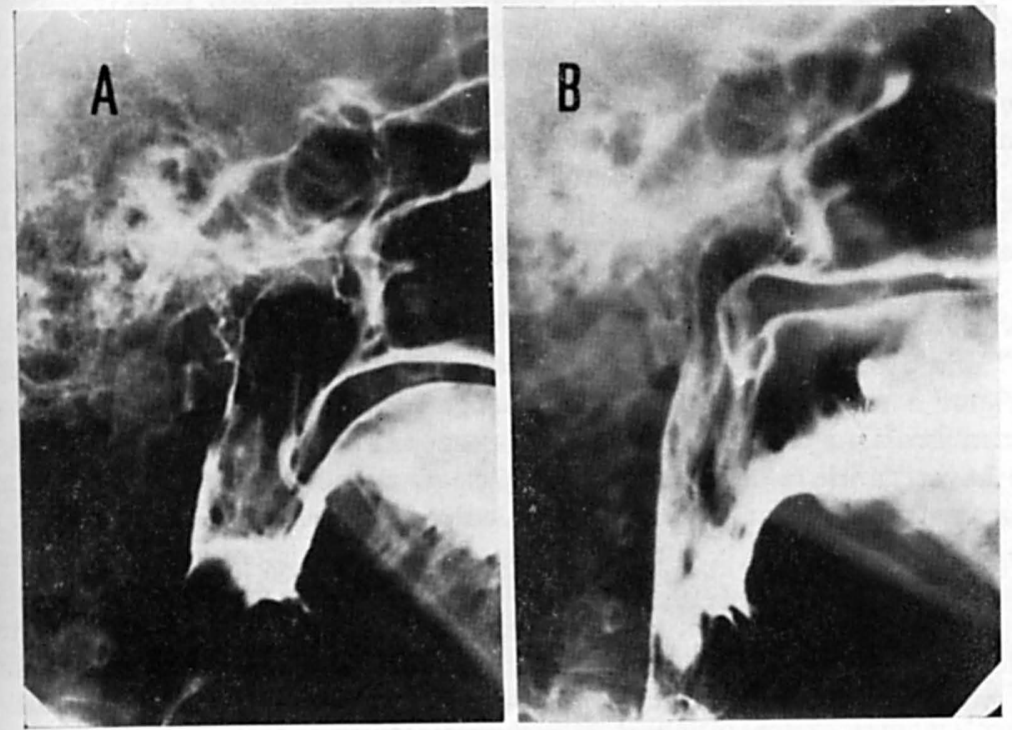

Fig. 2 - Caso V. V. C. Trânsito esofagiano mostrando refluxo faringo-nasal. 


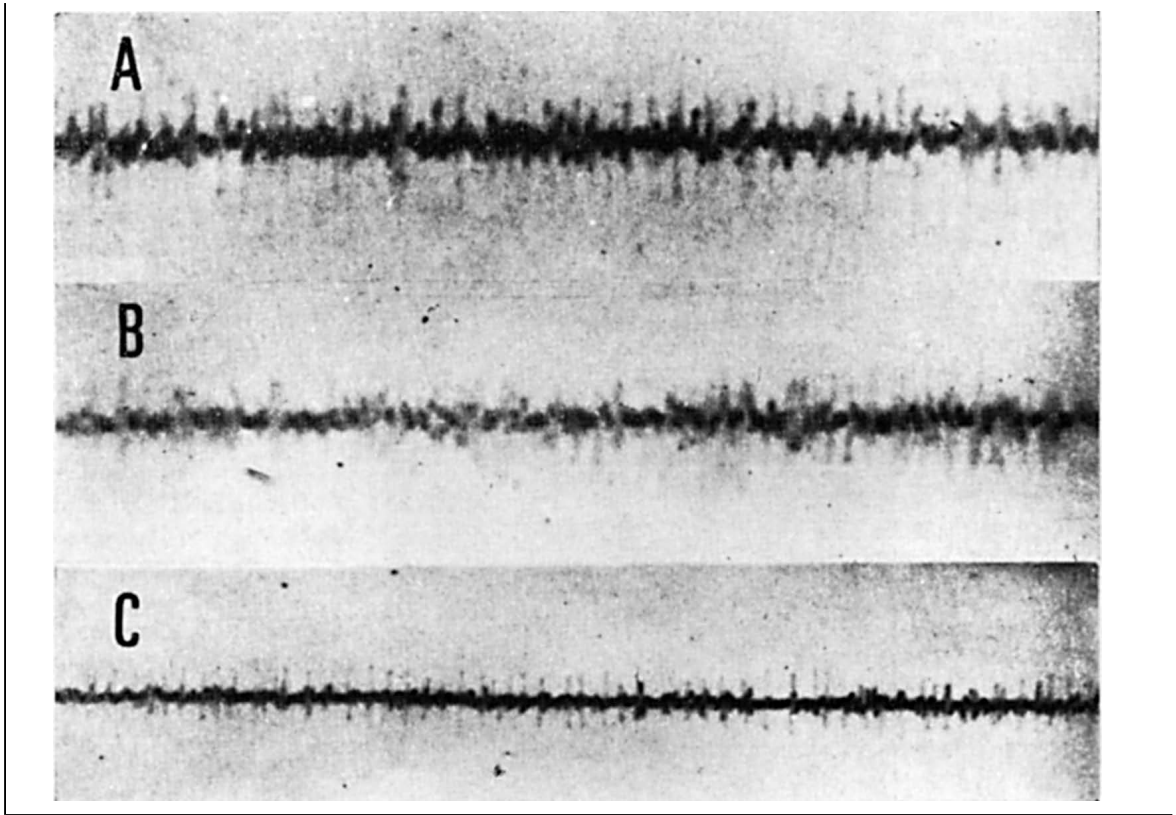

Fig. 3 - Caso V. V. C. Eletromiogramas: em A, músculo elevador da pálpebra D.; em $B$, músculo reto lateral $D$; em $C$, músculo masseter $D$. - Traçados eletromiográficos do tipo miogênico.

A primeira, individualizada por Kilih e Nevin, em 1951, caracteriza-se por ptose palpebral bilateral e paralisia dos músculos extrínsecos dos olhos, de evolução lenta e progressiva, tendo inicio, geralmente, antes da $3^{\mathbf{a}}$ década da vida, sendo de carater familial (autossômico dominante), em 50\% dos casos. Outros músculos podem ser comprometidos, mormente os orbiculares das pálpebras. Outros autores também estudaram tal miopatia (Fuchs ${ }^{7}$, Sandifer ${ }^{17}$, Gartner \& Billet ${ }^{8}$, Cameron ${ }^{5}$, Schwarz e Liu ${ }^{18}$, Nicolaissen e Brodal ${ }^{14}$, Boudien e col. ${ }^{3}$, Teasdall e Lears $^{20}$, Lundberg ${ }^{12}$ ).

A miopatia ocular descendente foi, primeiramente, estudada por Taylor ${ }^{19}$ (1915) que a atribuiu à degeneração dos núcleos do tronco cerebral e, depois, por Victor e col. ${ }^{21}$ e por Lees e Liversedge ${ }^{11}$ que a colocaram entre os processos musculares primários. Caracteriza-se por ptose palpebral bilateral, podendo haver paralisia dos músculos extrínsecos do olho e por paralisia da musculatura estriada da faringe, de evolver lento e progressivo. $\mathrm{O}$ início é em torno da $5^{a}$ década da vida e, em quase todos os casos, existem antecedentes familiares com o mesmo quadro. Geralmente, a paralisia dos músculos oculares inicia o cortejo, seguindo-se, mais tarde, o acometimento faringeo. A autonomia desta afecção é defendida por Bray e col. ${ }^{4}$ e Walton ${ }^{23,}{ }^{24}$, baseando-se no inicio tardio e no caráter autossômico dominante. Foi também estudada por Hayes e col. ${ }^{9}$, Peterman e col. ${ }^{15}$, Rebeix e col. ${ }^{16}$, Murphy e Drachman ${ }^{13}$, Aarli ${ }^{1}$, Finkel e col. ${ }^{6}$. 

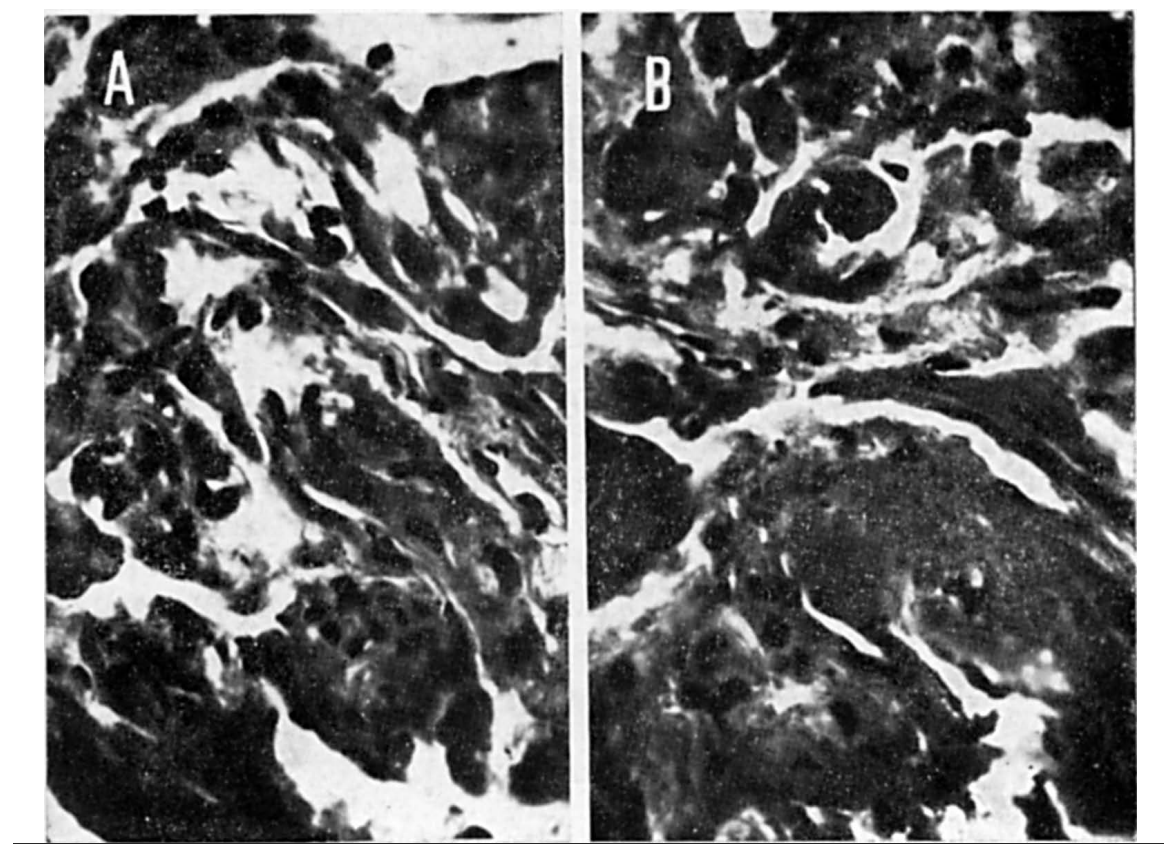

Fig. 4 - Caso V. V. C. Aspectos histológicos revelando fibras musculares atróficas, com diferentes diâmetros, e floculação do sarcoplasma (H.E. $24 X$ ): em A, músculo elevador da pálpebra $D . ; \mathrm{em} B$, músculo reto lateral D.

O discrime diferencial com a miastenia grave se impõe. Na miastenia, além da história de piora dos sintomas no decorrer do dia, na maioria dos casos, há regressão da sintomatologia com o teste da prostigmina. Apesar disto, em certas formas crônicas desta afecção da placa motora, podemos encontrar paralisias irreversíveis pela atrofia muscular que se instala.

A paciente em lide, pelo comprometimento muscular ocular e faríngeo, apresentaria a chamada miopatia ocular descendente; o traçado eletromiográfico somado à histopatologia muscular comprovam o diagnóstico de miopatia primária. Todavia, o início precoce e a inexistência de casos semelhantes na familia fugiram às características primordiais desta afecção (Bray e col. ${ }^{1}$, Walton ${ }^{23},{ }^{24}$ ). Entretanto, nas primeiras observações de Kiloh e Nevin ${ }^{10}$, já era feita menção ao acomentimento de músculos inervados pelo segmento bulbar em $10 \%$ dos casos. Acreditamos, assim, que não se justificaria a subdivisão das miopatias oculares em duas entidades autônomas e sim que seriam variantes clínicas de uma mesma afecção que acometeria primeiramente os músculos oculares, podendo acometer outros músculos, principalmente os responsáveis pela deglutição e fonação. 


\section{RESUMO}

Os autores apresentam caso de paciente jovem, do sexo feminino, com afecção muscular primária ocular e faríngea sem caráter familial. Foram feitos estudos eletromiográficos e histopatológicos musculares que confirmam o carater miogênico do processo. É feita comparação entre a miopatia ocular e a miopatia ocular descendente, acreditando os autores que seriam variantes de uma mesma afecção muscular.

\section{SUMMARY}

\section{Descending oculur myoputhy: a cuse report}

The case of a 23 years old female patient, with primary involvement of the extraocular and faringeal muscles without familiar history is reported. Electromyographic and muscular biopsy studies proved the myogenic nature of the process. A clinical comparison between the ocular myopathy and the descending ocular myopathy is made, the authors thinking that both of them would be variants of the same muscle disease.

\section{REFERENCIAS}

1. AARLI, J. A. - Oculopharyngeal muscular dystrophy. Acta Neurol. Scandinav. 45:484, 1969.

2. BARBEAU, A. - Oculopharyngeal muscular dystrophy in French. Canada. Trans. II Int. Congr. Neuro-Genetics and Neuro-Ophthalmology (Montreal), 1967.

3. BOUDIN, G.; PÉPIN B. \& AUFFRET, M. - A propos de trois cas de myopathie oculaire. Rev. Neurol. (Paris) 102:173, 1960.

4. BRAY, G. M.; KAARSO, M. \& ROSS, R. T. - Ocular myopathy with dysphagia. Neurology (Minneapolis) 15:678, 1965.

5. CAMERON, P. B. - Familial ptosis of the eyelids appearing in middle life. Arch. Ophthal. (Chicago) 43:818, 1950.

6. FINKEL, N.; RIBEIRO, A. R. V. \& SAMPAIO, M. C. R. - Miopatia ocular descendente. Arq. Neuro-Psiquiat. (São Paulo). 30:148, 1972

7. FUCHS, E. - Uber isolierte doppelseitige Ptosia. Arch. für Ophth. 36:234, 1890.

8. GARTNER, S. \& BILLET, E. - Progressive muscular dystrophy involving the extraocular muscles. Arch. Ophthal. (Chicago). 41:334, 1949.

9. HAYES, R.; LONDON, W. LEIDMAN, J. EEMBREE, L. - Oculo-pharyngeal muscular dystrophy. New Engl. J. Med. 268:163, 1963.

10. KILOH, L. G. \& NEVIN, S. - Progressive dystrophy of the external ocular muscles (ocular myopathy). Brain 74:115, 1951.

11. LEES, F. \& LIVERSEDGE, L. A. - Descending ocular myopathy. Brain 85:701, 1962.

12. LUNDBERG, P. O. - Ocular myopathy with hipogonadism. Acta Neurol. Scandinav. $38: 142,1962$.

13. MURPHY, S. F. \& DRACHMAN, D. B. - The oculopharingeal syndrome, J. A. M. A. 203:1003, 1968 .

14. NICOLAISSEN, B. \& BRODAL, A. - Chronic progressive external ophthalmoplegia. Arch. Ophthal. (Chicago). 61:202, 1959.

15. PETERMAN, A. F.; LILlingtoN, G. A. \& JAMPLis, R. W. - Progressive muscular dystrophy with ptosis and dysphagia. Arch. Neurol. (Chicago) 10:38, 1964.

16. REBEIX, J. J.; CAULFIELD, J. B. \& ADAMS, R. D. - A pathological study of oculopharyngeal dystrophy. Trans II Int. Congr. Neurogenetics and NeuroOphthalmology (Montreal), 1967. 
17. SANDIFER, P. H. - Chronic progressive ophthalmoplegia of myopathic origin. J. Neurol. Neurosurg. Psychiat. 9:81, 1946.

18. SCHWARZ, G. A. \& LIU, C. - Chronic progressive external ophthalmoplegia Arch. Neurol, Psychiat. (Chicago) 71:31, 1954

19. TAYLOR, E. M. - Progressive vagus-glossopharyngeal paralysis with ptosis. A contribuition to the group of family diseases. J. Nerv. Ment. Dis. 42:129, 1915.

20. TEASDALL, R. D. \& LEARS, M. L, - Ocular myopathy. Arch. Neurol. (Chicago) $2: 281, \quad 1960$.

21. VICTOR, M.; HAYES R. \& ADAMS, R. D. - Oculopharyngeal muscular dystrophy: a familial disease of late life characterized by dysphagia and progressfve ptosis of eyelids. New Engl. J. Med. 267:1267, 1962.

22. WALSH, F. B. - The eyelids and the extraocular muscles. In Clinical NeuroOphthalmology. The Williams \& Wilkins Co., Baltimore, 1957.

23. WALTON, J. N. - Progressive Muscular Dystrophy, In Disorders of Voluntary Muscles. Little, Brown Co., Boston, 1969.

24. WALTON, J. N. \& PERMINGTON, R. J. T. - Studies on human muscular dystrophy with particular reference to methods of carrier detection. An. N. Y. Acad. Sci. 138:315, 1966.

Instituto de Neurologia Deolindo Couto - Aveniala Venceslau Braz 95, Botatoyo - 20.00n Rio de Janeiro, GB - Brasil. 